\title{
CLASSIFYING OPTICAL PROPERTIES OF SURFACE- AND BULK-SCATTERING BIOLOGICAL LAYERS WITH POLARIZATION SINGULAR STATES
}

\author{
YU. A. USHENKO*, G. B. BODNAR and G. D. KOVAL \\ Correlation Optics Department, Chernivtsi National University \\ 2 Kotsyubinsky Str. Chernivtsi 58012, Ukraine \\ *yuriyu@gmail.com
}

Received 4 March 2013

Accepted 5 May 2013

Published 18 June 2013

\begin{abstract}
The results of singular approach usage in the tasks of description and classification of appearance of optical anisotropy of different types of phase-inhomogeneous biological layers (surfacescattering, optically thin and optically thick) have been presented. The characteristic values of the fourth Stokes vector parameter $\left(S_{4}=0\right.$ - linear polarization - ( $L$-state); $S_{4}= \pm 1-$ circular polarization - $( \pm C$-state $))$ have been chosen as the main analytical tool describing polarization-singular states. The value of $S_{4}$ has been determined by the value of phase shift between the orthogonal components of amplitude in the point of biological layer laser image and therefore is azimuthally stable. Hence, statistic moments of the first to the fourth orders characterizing the distribution of the amount of characteristic values $S_{4}=0 ; S_{4}= \pm 1$ have been used for definition and differentiation of optical properties of different types of biological layers surface scattering, optically thin and optically thick human skin.
\end{abstract}

Keywords: Polarization; birefringence; statistical optics; medical and biological imaging.

\section{Introduction}

By tradition, the processes of transforming optical radiation of phase-inhomogeneous objects and media are considered, as a rule, in a statistic approach (theory of radiation transfer, ${ }^{1-5}$ Monte-Carlo modeling $\left.{ }^{6,7}\right)$. Among the most spread traditional methods for studying the scattered light fields, one can separate the following independent directions: "scalar" (photometry and spectrophotometry) ${ }^{8-10}$ and "vector" (polarization nephelometry and Muellermatrix optics). ${ }^{11-15}$ Using these approaches, interrelations between the sets of statistic moments of the 1 st to the 4 th orders have been found. ${ }^{16,17}$

Compared with traditional statistic investigations, new optical approaches for describing the structures of fields in the case of scattered coherent radiation have been formed in recent $10-15$ years. The complex coordinate distribution of azimuths

This is an Open Access article published by World Scientific Publishing Company. It is distributed under the terms of the Creative Commons Attribution 3.0 (CC-BY) License. Further distribution of this work is permitted, provided the original work is properly cited. 
and ellipticities values, formed due to statistic interference of partial coherent waves, is typical for such fields. Hereinafter, we will call such fields "polarization-inhomogeneous ones". The main feature of this approach is the analysis of particular (boundary) polarization states to determine the whole structure of coordinate distributions for azimuths and ellipticities of polarization. The so-called polarization singularities are commonly used as these states ${ }^{18-22}$ :

- states with linear polarization when the direction of rotation for the electric field vector is indefinite, the so-called $L$-states;

- circularly polarized states when the azimuth of polarization for the electric field vector is indefinite, the so-called $C$-states.

It is shown in works ${ }^{23-27}$ that having the information about the coordinate networks of such points, it is possible to determine the peculiarities of the field's topological structure. Still, the data that has been obtained by now are generally of theoretical nature. It is noteworthy that practically all the variety of biological objects possesses the optical anisotropy, which reveals in formation of $L$ - and $C$-states networks.

Presently, there are no systematic data about polarization-singular structure of laser fields scattered by biological layers of different morphological structure and optical thickness. ${ }^{27}$ Besides, formation of networks of linearly and circularly polarized states is azimuthally dependent to changes of both probing beam polarization plane and investigated sample orientation. ${ }^{28}$ Therefore, there is a topical task of elaboration of universal technique of diagnostic and differentiation of optical properties of different biological layers within the singular approach with the help of different azimuthally stable parameters.

We have chosen the characteristic values of the fourth Stokes vector parameter, which universally describe all polarization-singular states as such parameter.

$$
\left\{\begin{array}{l}
L-\rightarrow S_{4}=0 \\
\pm C-\rightarrow S_{4}= \pm 1
\end{array}\right.
$$

Another important circumstance is that the value of $S_{4}$ is determined by the value of phase shift $\delta$ between the orthogonal components of amplitude in point of laser image of biological layer and, therefore, is azimuthally stable. That is why, the distributions $S_{4}=0(\delta=0)$ and $S_{4}= \pm 1(\delta= \pm 0,5 \pi)$ can be used as universal and azimuthally stable characteristic of optical properties of biological layers of different morphological structures.

This work is directed at investigation of possibility of definition and differentiation of optical properties of biological layers of different types surface-scattered, optically thin (single scattered) and optically thick (multiple scattered, depolarized) in the framework of statistic description of distributions $S_{4}=0$ and $S_{4}= \pm 1$.

\section{Model Conceptions and Analytical Relations}

As a basis for analytical description of the processes providing the formation of polarization-inhomogeneous images for various types of biological phaseinhomogeneous layers (PhIL), we have used the model concepts developed in the works ${ }^{27,28}$ :

1. Four main types of tissues - connective, muscular, epithelial and neural - represent the variety of human biological tissues.

2. Optically, morphological structure of any biological tissue type is considered as a superposition of phase-inhomogeneous surface (rough) and volume (isotropic-anisotropic) scattered components.

3. Surface-scattering PhIL (attenuation coefficient $\tau \leq 0.1$ ) is a rough surface (superficial layer of the skin epithelium) consisting of the ensemble of quasi plane, randomly tilted micro-areas with optical dimensions $l>\lambda-$ group 1. The optical properties of a local micro-area of such surface are described by the following matrix operator $\{R\}$ :

$$
\begin{aligned}
\{R\}= & \left(r_{x}^{2}+r_{y}^{2}\right)^{-1} \\
& \times \|\left(\begin{array}{cccc}
\left(r_{x}^{2}+r_{y}^{2}\right) & \left(r_{x}^{2}-r_{y}^{2}\right) & 0 & 0 \\
\left(r_{x}^{2}-r_{y}^{2}\right) & \left(r_{x}^{2}+r_{y}^{2}\right) & 0 & 0 \\
0 & 0 & \left(2 r_{x} r_{y}\right) & 0 \\
0 & 0 & 0 & \left(2 r_{x} r_{y}\right)
\end{array} \|,\right.
\end{aligned}
$$

where $r_{x}(\gamma, n), r_{y}(\gamma, n)$ are the Fresnel amplitude coefficients of reflection for orthogonal $(x, y)$ components of laser wave amplitude; $\gamma$ is the tilt angle of micro-area with respect to macro-surface; $n$ is the epithelium refractive index. 
4. Optically thin (attenuation coefficient $\tau \leq 0.1$ ) single scattered biological PhIL with anisotropic component can be described by the Mueller matrices for circular (optical activity) $\{V\}$ and linear (linear birefringence) $\{W\}$ phase

$$
W=\| \begin{array}{cc}
1 & 0 \\
0 & \cos ^{2} 2 \rho+\sin ^{2} 2 \rho \cos \delta \\
0 & \cos 2 \rho \sin 2 \rho(1-\cos \delta) \\
0 & \sin 2 \rho \sin \delta
\end{array}
$$

Here, $\rho$ is the direction (orientation azimuth) of fast optical axis; $\delta=2 \pi / \lambda \Delta n d$ is the value of linear phase anisotropy, phase shift between orthogonally linearly polarized components of laser amplitude $(\delta \in[0 ; \pi]) ; \lambda$ is the wavelength; $d$ is the geometric path; $\Delta n$ is the index of birefringence; $\theta$ is the value of circular phase anisotropy (rotation angle of polarization plane of laser radiation), $\theta \in[0 ; \pi]$.

5. For optically thick (attenuation coefficient $\tau>0.1$ ) multiple scattered biological PhIL group 3 - the main mechanism of local polarization state formation is interferometric superposition of amplitudes of coherent waves with different polarization. The result of such superposition reveals the formation of elliptical polarization described by the following equation

$$
\frac{X^{2}}{U_{x}^{2}}+\frac{Y^{2}}{U_{y t}^{2}}-2 \frac{X Y}{U_{x} U_{y}}=\sin ^{2} \varphi
$$

where $U_{x}, U_{y}$ are the orthogonal components of amplitude $U, \varphi$ is the phase shift between them.

Let us consider, in the framework of vectorparametric approach, the process of polarizationsingular states formation $\left(S_{4}=0 ; \pm 1\right)$. In the common case, the polarization transformation processes can be described using the following vectorparametric equation

$$
S^{*}=\{M\} S_{0},
$$

where $S_{0}, S^{*}$ are the Stokes vectors of illuminated and are transformed by the objects beam correspondingly; $\{M\}$ is the Mueller matrix of biological layer.

Using the matrix Eq. (6), with operators (2)-(4) structure and linear state of probing laser beam polarization $\left(S_{0}=\left(\begin{array}{c}1 \\ \cos 2 \alpha_{0} \\ \sin 2 \alpha_{0} \\ 0\end{array}\right)\right)$, where $\alpha_{0}$ is azimuth, it is possible to determine the conditions of formation anisotropy - group 2:

$$
V=\left\|\begin{array}{cccc}
1 & 0 & 0 & 0 \\
0 & \cos 2 \theta & \sin 2 \theta & 0 \\
0 & -\sin 2 \theta & \cos 2 \theta & 0 \\
0 & 0 & 0 & 1
\end{array}\right\|
$$

$$
\begin{array}{cc}
0 & 0 \\
\cos 2 \rho \sin 2 \rho(1-\cos \delta) & -\sin 2 \rho \sin \delta \\
\sin ^{2} 2 \rho+\cos ^{2} 2 \rho \cos \delta & \cos 2 \rho \sin \delta \\
-\cos 2 \rho \sin \delta & \cos \delta
\end{array} \| .
$$

of linear $\left(S_{4}=0\right)$ and circular $\left(S_{4}= \pm 1\right)$ polarization-singular states of the images of groups 1-3 objects:

\section{Group 1}

There are only $L$-states $\left(S_{4}=0\right)$ forming in this case irrespective of Fresnel coefficients $r_{x}(\gamma, n)$, $r_{y}(\gamma, n)$ variations, which are determined by refractive index $n$ and tilts $\gamma$ of quasi-planar epithelium plates.

\section{Group 2}

- Circular phase anisotropy

There are only $L$-states $\left(S_{4}=0\right)$ forming in this case irrespective of the value of phase shift between the circularly polarized components of amplitude of laser radiation transformed by optically active structures (3) of biological layer.

- Linear phase anisotropy

There are $L$-states $\left(S_{4}=0\right)$ forming on the condition of coincidence of probing beam polarization plane $\alpha_{0}$ and direction of optical axis $\rho$ of birefringent fibril

$$
\rho=\alpha_{0} .
$$

There are $\pm C$-states $\left(S_{4}= \pm 1\right)$ forming on the condition

$$
\rho=\alpha_{0} \pm 0.25 \pi ; \quad \delta= \pm 0.5 \pi .
$$

\section{Group 3}

Polarization-singular states in the field of multiple scattered laser radiation (5) are determined by the conditions

$$
\begin{gathered}
U_{x}=U_{y} ; \\
\varphi= \pm 0.5 \pi .
\end{gathered}
$$

Thus, we have demonstrated the basic interconnections between the optical-geometric structure of different types of biological layers and mechanisms of polarization-singular states formation. 


\section{Experimental Setup for Polarimetric Investigation}

Our study of polarization-inhomogeneous laser images inherent to PhIL was performed using the optical scheme of a laser polarimeter (see Fig. 1). ${ }^{27}$

The object of investigation was illuminated by collimated $\left(\varnothing=10^{4} \mu \mathrm{m}\right) \mathrm{He}-\mathrm{Ne}$ laser beam $(\lambda=$ $0.6328 \mu \mathrm{m})$ with the power of $50 \mu \mathrm{W}$. Polarization light source (quarter-wave plates 3,5 and polarizer 4 ) formed the ensemble of Stokes vectors of the illuminating beam $\left\{S_{j=1,2,3,4}^{0}\right\}$. By means of micro-objective 7 (focal distance $-1.5 \mathrm{~cm}$, aperture - 0.2, magnification - 4x), the polarization images of biological tissue were projected into the plane of light-sensitive area of CCD camera (total amount of pixels $800 \times 600$, light-sensitive area size $-4000 \times$ $3000 \mu \mathrm{m}$, deviation of photosensitive characteristic from linear one - no more than 15\%), which provided the range of measuring the structural elements of biological tissue with the resolution $2-2000 \mu \mathrm{m}$. Maximal resolution verification $(2 \mu \mathrm{m})$ was performed using the stage micrometer (linear scale), the image of which was projected into the light-sensitive area of CCD camera with the help of micro-objective 7. Minimal resolution $(2000 \mu \mathrm{m})$ corresponds to the situation, when the light-sensitive area of CCD camera is entirely filled by two equal-sized structural elements (light and dark) of stage micrometer. The conditions of the experiment were chosen in such a way that it enabled to reduce the space-angular aperture filtering while forming the biological tissue images. This was ensured by conformance of angular characteristics of indicatrices of light scattering by the biological tissue samples $\left(\Omega \approx 16^{\circ}\right)$ and angular aperture of micro-objective $\left(\Delta \omega=20^{\circ}\right)$. Here, $\Omega$ is the solid angle within which $98 \%$ of all the energy of light-scattered radiation is concentrated.

The technique of measuring the number of polarization-singular states in the plane of image of biological layers consists of the following steps:

- Turning the transmission axis of analyzer 9 by the angles $\pm 45^{\circ}$ relative to the direction of the highest velocity axis for the quarter-wave plate 8, we determined the intensities of right $\left(I_{\otimes}\right)$ and left $\left(I_{\oplus}\right)$ circularly polarized components for each separated pixel $\left(r_{i k}\right)$ of CCD camera 10.

- It served as a base to calculate the coordinate distributions of the fourth parameter in the Stokes vector $V_{4}(m \times n)$ describing the laser image of PhIL, using the relation

$$
V_{4}\left(r_{m n}\right)=\frac{I_{\otimes}\left(r_{i k}\right)-I_{\oplus}\left(r_{i k}\right)}{I_{\otimes}\left(r_{i k}\right)+I_{\oplus}\left(r_{i k}\right)} .
$$

- The two-dimensional array (11) was scanned along the horizontal direction $x \equiv 1, \ldots, m$ with the step $\Delta x=1$ pix.

- Within the limits of each local sample $\left(1_{\text {pix }} \times\right.$ $\left.n_{\text {pix }}\right)^{(k=1,2, \ldots, m)}$, we calculated the amount $(N)$ of characteristic values $V_{4}(k)=0,-\left(N_{L}^{(k)}\right)$ and $V_{4}(k)= \pm 1,-\left(N_{ \pm C}^{(k)}\right)$.

Thus, we determined the dependences $N_{L}(x) \equiv$ $\left(N_{L}^{(1)}, N_{L}^{(2)}, \ldots, N_{L}^{(m)}\right)$ and $N_{ \pm C}(x) \equiv\left(N_{ \pm C}^{(1)}, N_{ \pm C}^{(2)}\right.$, $\left.\ldots, N_{ \pm C}^{(m)}\right)$ for the sets of polarization-singular $L$ and $\pm C$-points within the limits of a laser image for PhIL.

For quantitative assessment of the following distributions $N_{L}(x) \equiv\left(N_{L}^{(1)}, N_{L}^{(2)}, \ldots, N_{L}^{(m)}\right)$ and $N_{ \pm C}(x) \equiv\left(N_{ \pm C}^{(1)}, N_{ \pm C}^{(2)}, \ldots, N_{ \pm C}^{(m)}\right)$ in this work we have used the statistic approach. ${ }^{28}$ Distributions $N_{L, \pm C}(x)$ for the set of polarization-singular states in laser images of PhIL are characterized by the ensemble of statistic moments of the 1st-4th orders $Z_{j=1,2,3,4}$ calculated using the following relations

$$
\begin{aligned}
Z_{1} & =\frac{1}{M} \sum_{i=1}^{M}\left|N_{L, \pm C}^{(i)}(x)\right|, \\
Z_{2} & =\sqrt{\frac{1}{M} \sum_{i=1}^{M}\left[N_{L, \pm C}^{(i)}(x)-Z_{1}\right]^{2}}, \\
Z_{3} & =\frac{1}{Z_{2}^{3}} \frac{1}{M} \sum_{i=1}^{M}\left[N_{L, \pm C}^{(i)}(x)\right]^{2},
\end{aligned}
$$

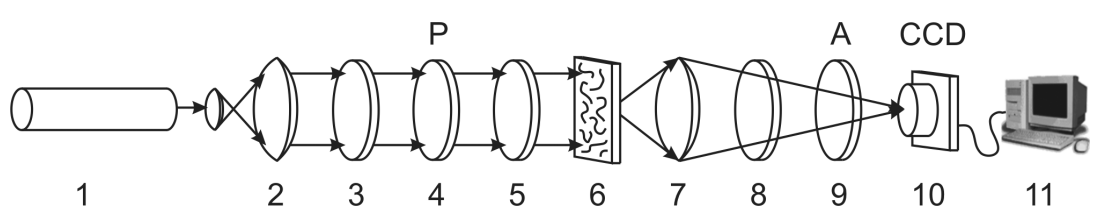

Fig. 1. Optical scheme of the polarimeter: 1 - He-Ne laser; 2 - collimator; $3,5,8$ - quarter-wave plates; 4, 9 - polarizer and analyzer, respectively; 6 - object of investigation; 7 - micro-objective; 10 - CCD camera; 11 - personal computer. 


$$
Z_{4}=\frac{1}{Z_{2}^{4}} \frac{1}{M} \sum_{i=1}^{M}\left[N_{L, \pm C}^{(i)}(x)\right]^{4},
$$

where $M=800 \times 600$ is the amount of pixels in CCD camera 10 (see Fig. 1).

\section{The Investigation Objects Characteristics}

The following types of histological sections of skin dermis samples taken from the human stomach region were used as the objects of investigation:

- superficial optically thin (the attenuation coefficient $\tau=0.085$ ) epidermis layer, which is formed by the set of keratinized isotropic epithelium plates - group 1;

- optically thin $(\tau=0.095)$ dermis layer, where the anisotropic component is formed by a network of collagen birefringent fibrils - group 2;

- optically thick $(\tau=0.45)$ dermis layer - group 3 .

The histological sections were prepared with the standard technique by freezing microtome. They have the following characteristics: the absorption coefficient $\mu_{a}=2.2 \mathrm{~cm}^{-1}$, the scattering coefficient $\mu_{s}=185 \mathrm{~cm}^{-1}$, the anisotropy parameter $g=0.82$, value of birefringence $\Delta n(S D) \approx 1.45 \times 10^{-3}$, the geometric thickness $d=15 \mu \mathrm{m} \quad(\tau=0.095)$ и $d=$ $40 \mu \mathrm{m}(\tau=0.45)$.

Polarization manifestations of peculiarities of morphological structure are presented by coordinate $(100 \times 50$ pix $)$ distributions of the fourth parameter for the Stokes vector $V_{4}(m \times n)$ inherent to laser images of PhIL in all the groups (see Fig. 2).

Our qualitative analysis of coordinate distributions $V_{4}(m \times n)$ for laser images of PhIL (see
Fig. 2) enabled to reveal that:

- practically all the images of the rough surface of skin [see Fig. 2(a)] are a linearly polarized field $V_{4}(m \times n)=0$. This fact is in good agreement with the performed modeling (relations (2) and (6)). Availability of a small amount of the parts $V_{4}(m \times n) \neq 0$ polarized otherwise can be related to the interference effects of multiple-scattered coherent waves with adjacent micro-roughnesses;

- the image of the optically thin layer of the dermis [see Fig. 2(b)] is characterized with a developed polarization-inhomogeneous structure formed both by linearly $\left(V_{4}(m \times n)=0\right.$ - caused by mechanisms of circular phase anisotropy or optical activity (relations (7)) and elliptically $\left(V_{4}(m \times n) \neq 0\right)$ polarized states, including the circularly $\left(V_{4}(m \times n)=1\right)$ polarized ones caused by the mechanisms of linear phase anisotropy or linear birefringence [relations (8)];

- the images of the optically thick layer of skin [see Fig. 2(c)] are characterized with the widest range of changing azimuth and ellipticity of polarization $\left(-1 \leq V_{4}(m \times n) \leq 1\right)$ formed by two groups of mechanisms. The first one is the linear and circular phase anisotropy of collagen fibrils (9), (10). The second one is the interference of differently polarized partial fronts and the formation of polarization-inhomogeneous speckle field.

\section{Experimental Results and Discussion}

\subsection{L-states of laser images}

The series of coordinate $\left(V_{4}(m \times n)=0\right)$ and quantitative $\left(N_{L}(x)\right)$ distributions for polarizationsingular $L$-states in laser images of PhIL is summarized in Fig. 3.

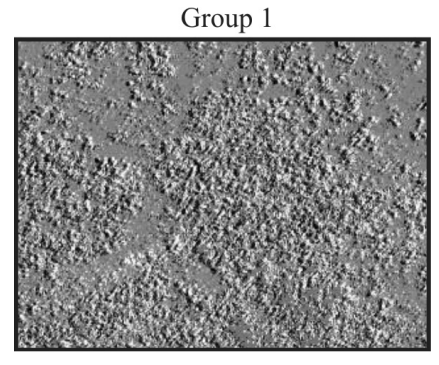

(a)

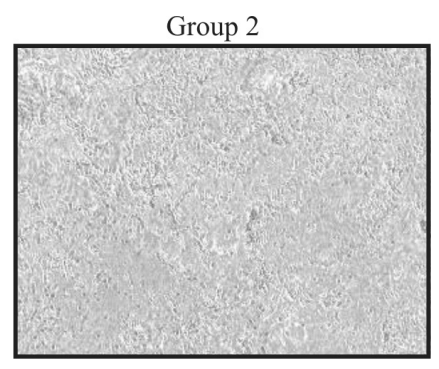

(b)

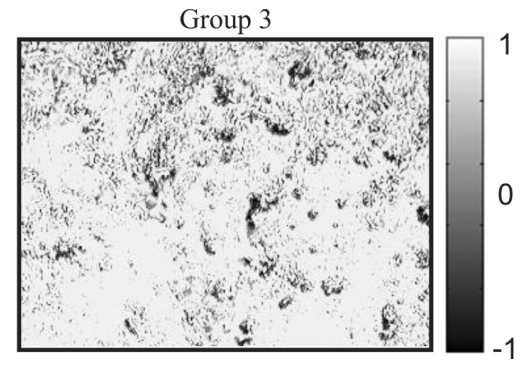

(c)

Fig. 2. Coordinate distributions of $V_{4}(m \times n)$ of laser images inherent to PhIL. 


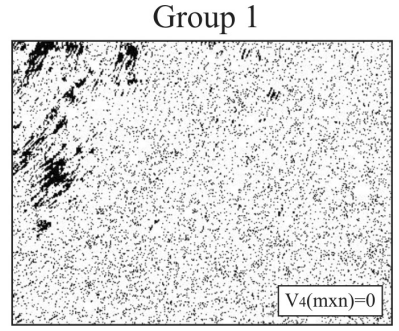

(a)

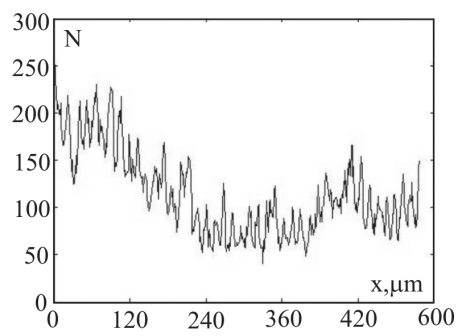

(d)

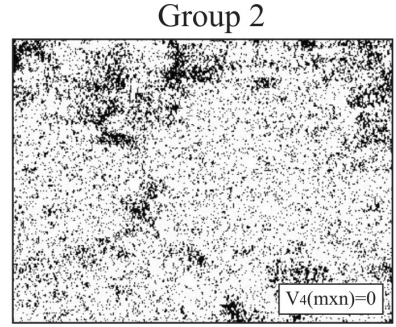

(b)

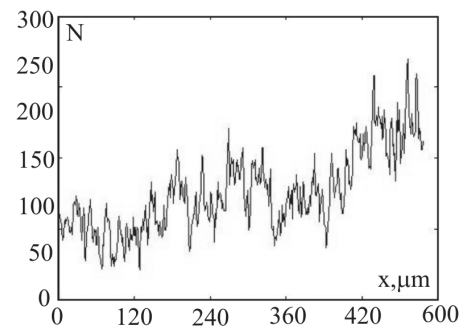

(e)

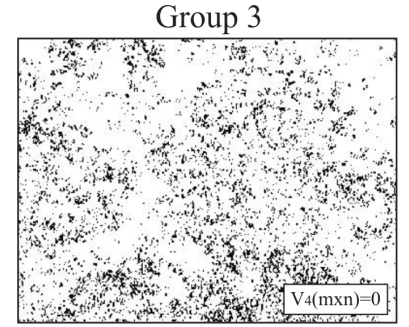

(c)

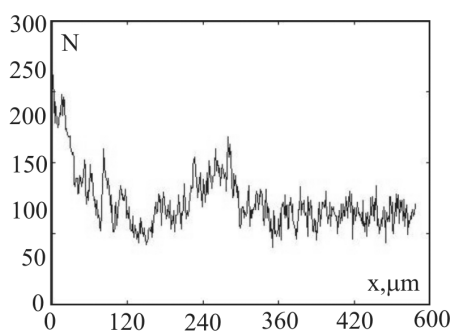

(f)

Fig. 3. Coordinate $V_{4}(m \times n)=0$ (fragments a, b, c) and quantitative $N_{L}(x)$ distributions (fragments d, e, f) of $L$-states in polarization for laser images of the PhIL.

Our comparative analysis of the obtained dependences $N_{L}(x)$ for the set of polarizationsingular $L$-states [see Figs. $3(\mathrm{~d})-3(\mathrm{f})]$ in laser images of all groups of PhIL [see Figs. 3(a)-3(c)] in the framework of statistic approach revealed the following.

In the case of surface-scattering epidermis layer, the main mechanisms of transformation of polarization state are the local reflection (refraction) acts by keratinized, optically isotropic epithelium plates. Such interaction acts lead to the formation of polarization plane rotation, the value of which is determined by the tilt angle of microasperity and refraction index of matter. Therefore, the amount of $L$-states of polarization in the corresponding laser image appears to be dominant [see Figs. 3(a) and $3(\mathrm{~d})]$.

In the case of optically thin dermis layer, the simultaneous realization of two interaction mechanisms occurs. This results in the formation of both $L$-states (circular phase anisotropy (7) and $C$-states (linear phase anisotropy $\left.\delta=(2 q+1) \frac{\pi}{2},(8)\right)$. Due to this fact, the amount of $L$-states in laser image of such a layer decreases [see Figs. 3(b) and 3(e)]. Statistically, such a process is displayed in the decrease of the first statistic moment of $N_{L}(x)$ distribution and, vice versa, in the increase of the second statistic moment.

As for multiple-scattered skin dermis layer, the phase modulation, which arises due to linear phase anisotropy with subsequent interference of partial coherent waves, appears to be the prevailing mechanism of formation of local polarization states. Such a mechanism optically reveals in the essential increase of probability of elliptically polarized states formation (including $C$-states) under the simultaneous decrease of the total amount of $L$-states [see Figs. 3(c) and 3(f)]. Probably, the processes of multiple scattering of polarized laser radiation by the optically anisotropic collagen network reveal in subsequent decrease (increase) of the value of the first (second) statistic moments, which characterize the $N_{L}(x)$ distribution for the group 3 objects.

Quantitatively specified regularities of $L$-states formation in laser images of biological layers of all groups reveal the following facts.

The values of high-order statistic moments, which characterize the distributions of linearly polarized states $N_{L}(x)$, are considerably small in comparison with the statistic moments of the 1st2nd orders $-Z_{j=3,4}^{L} \ll Z_{j=1,2}^{L}$.

The distinctions between the distributions of $L$-states in laser images of various PhIL, which are observed as variations of the 1st and 2nd statistic moments $-Z_{1}^{L}=0.62, Z_{2}^{L}=0.105$ (group 1); $Z_{1}^{L}=0.39, Z_{2}^{L}=0.19\left(\right.$ group 2) and $Z_{1}^{L}=0.19$, $Z_{2}^{L}=0.28$ (group 3) can be used as classification and diagnostic parameters of object optical properties. 
Apparently, for PhIL of the 1st, 2nd and 3rd groups, the mean value $Z_{1}^{L}$ is by 1.5 and 3.3 times decreased. In addition, vice versa, the dispersion $Z_{2}^{L}$ is by 1.5 and 2.5 times increased.

\section{2. \pm C-states of laser images}

Summarized in Fig. 4 is the series of coordinate $\left(V_{4}(m \times n)=1\right)$ and quantitative $\left(N_{ \pm C}(x)\right)$ distributions for polarization-singular $\pm C$-states in laser images of PhIL. The absence of $C$-points in the image of the object of group 1 is designated as "- absent" in fragments (a, d).

The comparative analysis of data presented in Fig. 4 with the parameters of $L$-points networks (see Fig. 3) reveals sufficient distinctions between them.

In the laser image of the group $1, \pm C$-states of polarization are absent (see Fig. 4, left column fragments a, d), which once again confirms the adequacy of model conceptions of mechanisms providing transformation of laser radiation by the set of chaotically oriented micro-areas of the rough surface [relation (2)].

Optical manifestations of the anisotropic layer of collagen fibrils are illustrated by the network of $\pm C$-points in the laser image [see Fig. 4(b)]. One can see that for laser images of optically thin skin dermis layer (group 2) there is a considerabe large amount of $C$-points. This fact is the evidence of sufficiently probable forming of the right- and left-circulated polarization components of laser radiation transformed by the set of birefringent collagen fibers [relations (7), (8)].

In the framework of statistic approach, it is established that all the statistic moments $Z_{j=1,2,3,4}^{ \pm C}$ (relation (12)) that characterize the $N_{ \pm C}(x)$ distribution [see Figs. 4(b) and 4(e)] of the amount of circularly polarized singular states differ from zero: $Z_{1}^{ \pm C}=0.09, Z_{2}^{ \pm C}=0.33, Z_{3}^{ \pm C}=0.48$ and $Z_{4}^{ \pm C}=0.52$.

The comparison of the data obtained with the statistic moments values, which characterize the linear polarization states distributions, enables to reveal the sufficient increase in the values of the 3rd and the 4 th statistic moments.

The phase modulation of multiple scattered laser radiation by the optically thick layer of skin dermis (group 3) is characterized by the 〈most developed $\rangle$ network of $\pm C$-states [see Fig. 4(c)]. In this case, the total amount of $L$ - and $\pm C$-states of polarization for the corresponding laser image is approximately the same [see Figs. 3(f) and 4(f)]. This fact is, apparently, conditioned by the equiprobable presence of both mechanisms: statistic interference formation of

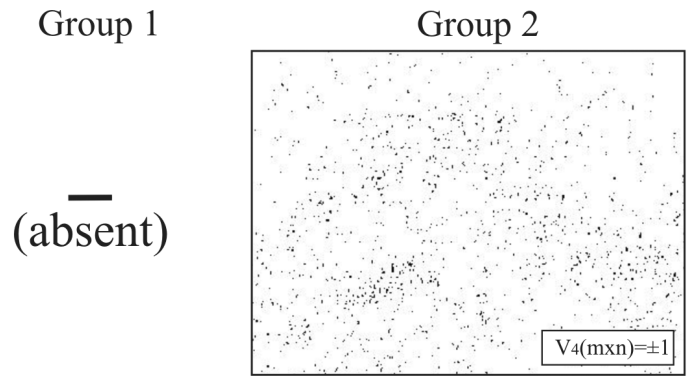

(a)

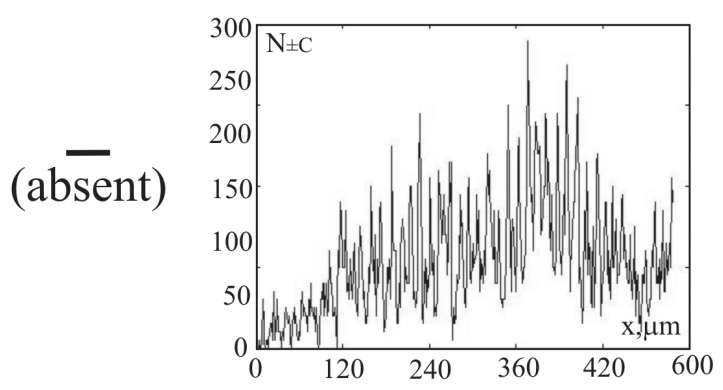

(d) (b)

(e)

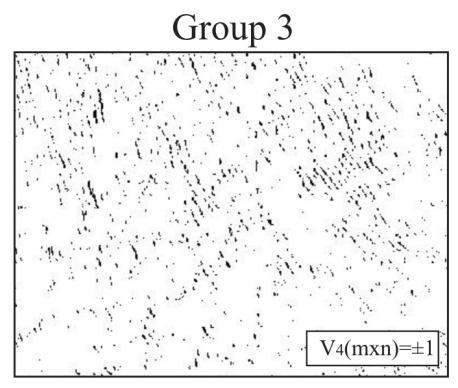

(c)

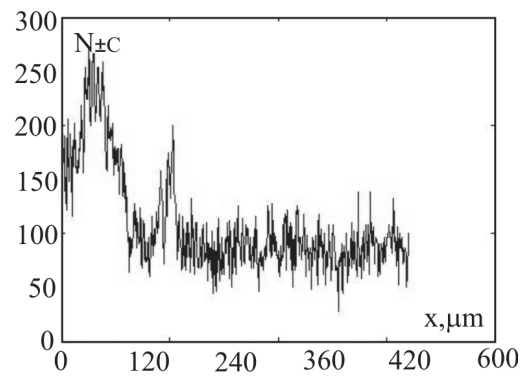

(f)

Fig. 4. Coordinate $V_{4}(m \times n)=1$ (fragments b, c), and quantitative $N_{ \pm C}(x)$ distributions (fragments e, f) of $\pm C$-states in polarization for laser images of PhIL. 
$L-(\varphi=q \pi, q=0,1,2 \ldots)$ as well as $\pm C-(\varphi=$ $\left.\pm(2 q+1) \frac{\pi}{2}, q=0,1,2 \ldots\right)$ states (relations (8)). The comparative analysis of $N_{L}(x)$ [see Figs. 3(c) and 3(f)] and $N_{ \pm C}(x)$ [see Figs. 4(c) and 4(f)] distributions detects its similarity. Thus, difference between statistic moments $Z_{j=1,2,3,4}^{L}$ and $Z_{j=1,2,3,4}^{ \pm C}$ is insignificant and lies within $25 \%$ to $35 \%$.

On the other hand, there exist the pronounced intergroup distinctions between the statistic [see Figs. 4(d)-4(f)] structures of $N_{ \pm C}(x)$ distributions in laser images of all groups [see Figs. 4(a)-4(c)]. This fact represents the background for not only the classification, but also differentiation of biological PhIL optical properties.

\section{Polarization-Singular Classification and Differentiation of Optical Properties Inherent to PhIL}

In order to define the possibilities of classification and differentiation of all types of biological layers, a comparative investigation of distributions of polarization singularities amount of laser images within the reliable amount of histological sections samples was performed. The histological sections of dermis samples were obtained from the autopsied material of 21 corpses. The statistical validity of the sampling is proved by confidence interval for each group $p \leq 0.01$.

The statistically averaged (within the limits of groups 1 to 3 ) values and ranges of changing statistic moments $Z_{j=1 ; 2 ; 3 ; 4}^{L ; \pm C}$ that characterize the $N_{ \pm C}(x)$ dependences for the amount of singular states in laser images of PhIL have been illustrated in Tables 1 and 2 .

The performed analysis of the results presented in Tables 1 and 2 for statistic $\left(Z_{j=1,2,3,4}^{L, \pm C}\right)$ parameters shows:

- the signature of predominance of surface scattering reveals in minimal values of statistic moments

Table 1. Statistic parameters for the distribution of the amount of $L$-states in laser images of PhIL.

\begin{tabular}{cccc}
\hline $\begin{array}{c}\text { Parameters } \\
(L \text {-states })\end{array}$ & $\begin{array}{c}\text { Group 1 } \\
(21 \text { samples })\end{array}$ & $\begin{array}{c}\text { Group 2 } \\
(21 \text { samples })\end{array}$ & $\begin{array}{c}\text { Group 3 } \\
(21 \text { samples })\end{array}$ \\
\hline$Z_{1}$ & $0.67 \pm 0.079$ & $0.43 \pm 0.045$ & $0.21 \pm 0.035$ \\
$Z_{2}$ & $0.11 \pm 0.034$ & $0.19 \pm 0.034$ & $0.29 \pm 0.045$ \\
$Z_{3}$ & $0.05 \pm 0.008$ & $0.07 \pm 0.009$ & $0.05 \pm 0.007$ \\
$Z_{4}$ & $0.11 \pm 0.044$ & $0.08 \pm 0.009$ & $0.08 \pm 0.03$ \\
\hline
\end{tabular}

Table 2. Statistic parameters for the distribution of the amount of $\pm C$-states in laser images of PhIL.

\begin{tabular}{cccc}
\hline $\begin{array}{c}\text { Parameters } \\
( \pm C \text {-states })\end{array}$ & $\begin{array}{c}\text { Group 1 } \\
(21 \text { samples })\end{array}$ & $\begin{array}{c}\text { Group 2 } \\
(21 \text { samples })\end{array}$ & $\begin{array}{c}\text { Group 3 } \\
(21 \text { samples })\end{array}$ \\
\hline$Z_{1}$ & - & $0.14 \pm 0.032$ & $0.21 \pm 0.041$ \\
$Z_{2}$ & - & $0.39 \pm 0.091$ & $0.26 \pm 0.034$ \\
$Z_{3}$ & - & $0.41 \pm 0.12$ & $0.08 \pm 0.01$ \\
$Z_{4}$ & - & $0.49 \pm 0.062$ & $0.07 \pm 0.05$ \\
\hline
\end{tabular}

of the third and the fourth orders, which characterize the distributions of $L$-states in the sensibe absence of $\pm C$-states;

- for optically thin anisotropic biological layer and for optically thick biological layers the tendency of formation of statistically similar distributions $L$ - and $\pm C$-states is the most typical;

- the differentiation of such types biological layers is possible using both the 1st and the 2 nd statistic moments, which characterize the distributions of $L$ - polarization states, and the $3 \mathrm{rd}$ and the 4 th statistic moments, which characterize the distributions of $\pm C$-polarization states;

- The difference between them reaches 1.5-2.6 $\left(Z_{1}\right)$ and 2-3 $\left(Z_{2}\right)$ times for $L$-states and $5\left(Z_{3}\right)-7\left(Z_{4}\right)$ times for $\pm C$-states.

\section{Conclusions}

From the results of performed investigations, one can conclude:

- The main mechanisms of formation of polarizationsingular states of laser images and interconnection with optical-geometric structure of surface, optically thin and optically thick skin derma layers have been determined.

- The universal, azimuthally stable technique of definition of polarization-singular structure of laser images of biological layers of different types using the statistic analysis of the distribution of the number of characteristic values of the fourth Stokes vector parameter $\left(S_{4}=0 ; \pm 1\right)$ has been elaborated.

- The values and the ranges of changes of statistic moments of the first to the fourth orders, which characterize the distributions of the number of characteristic values $\left(S_{4}=0 ; \pm 1\right)$ and can be used in diagnostics and differentiation of optical properties of surface, optically thin and optically thick skin derma layers have been defined. 


\section{References}

1. J. A. Moon, J. Reintjes, "Image resolution by use of multiply scattered light," Opt. Lett. 19, 521-523 (1994).

2. M. R. Ostermeyer, D. V. Stephens, L. Wang, S. L. Jacques, Nearfield polarization effects on light propagation in random media, in Biomedical Optical Spectroscopy and Diagnostics, E. Sevick-Muraca and D. Benaron, Eds., OSA Trends in Optics and Photonics Series, Vol. 3, Optical Society of America (1996), paper SP2.

3. V. Sankaran, M. J. Everett, D. J. Maitland, J. T. Walsh, Jr., "Comparison of polarized-light propagation in biological tissue and phantoms," Opt. Lett. 24, 1044-1046 (1999).

4. Y. Yasuno, S. Makita, Y. Sutoh, M. Itoh, T. Yatagai, "Birefringence imaging of human skin by polarization-sensitive spectral interferometric optical coherence tomography," Opt. Lett. 27, 18031805 (2002).

5. M. C. Pierce, J. Strasswimmer, B. H. Park, B. Cense, J. F. de Boer, "Birefringence measurements in human skin using polarization-sensitive optical coherence tomography," J. Biomed. Opt. 9, 287-291 (2004).

6. T. Yun et al., "Monte Carlo simulation of polarized light scattering in tissues," J. Innov. Opt. Health Sci. 2(2) 131-135 (2009).

7. X. Wang, L. V. Wang, "Propagation of polarized light in birefringent turbid media: A Monte Carlo study," J. Biomed. Opt. 7, 279-290 (2002).

8. R. R. Andersen, J. A. Parrish, Optical properties of the human skin, The Science of Photomedicine, J. A. Peagen, J. A. Parrish, Eds., pp. 147-193, Plenum Press, New York (1982).

9. M. J. C. Van Gemert, S. L. Jacques, H. J. C. M. Sterenborg, W. M. Star, "Skin optics," IEEE Trans. Biomed. Eng. 36, 1146-1154 (1989).

10. H. J. C. M. Sterenborg, M. J. C. van Gemert, W. Kamphorst, J. G. Wolbers, W. Hogervorst, "The spectral dependence of the optical properties of human brain," Lasers Med. Sci. 4, 221-227 (1989).

11. S. Jiao, G. Yao, L. V. Wang, "Depth-Resolved two-dimensional stokes vectors of backscattered light and mueller matrices of biological tissue measured with optical coherence tomography," Appl. Opt. 39, 6318-6324 (2000).

12. Q. Zhu et al., "Liquid crystal based rotating orthogonal polarization imaging system," J. Innov. Opt. Health Sci. 2(3), 245-251 (2009).

13. L.-P. Yu, "Polarization-sensitive optical coherence tomography using a modified balance detector," J. Innov. Opt. Health Sci. 5(4), 1250024 (2012).
14. O. V. Angel'skii, A. G. Ushenko, A. D. Arkhelyuk, S. B. Ermolenko, D. N. Burkovets, "Structure of matrices for the transformation of laser radiation by biofractals," Quantum Electron 29, 1074-1077 (1999).

15. A. G. Ushenko, "The vector structure of laser biospeckle fields and polarization diagnostics of collagen skin structures," Laser Phys. 10(5), 1143-1149 (2000).

16. O. V. Angel'skiı̌, A. G. Ushenko, A. D. Arkhelyuk, S. B. Ermolenko, D. N. Burkovets, Y. A. Ushenko, "Laser polarimetry of pathological changes in biotissues," Optika i Spectroskopiya 89(6), 10501055 (2000).

17. O. V. Angel'skiı̌, A. G. Ushenko, A. D. Arkhelyuk, S. B. Ermolenko, D. N. Burkovets, "Scattering of laser radiation by multifractal biological structures," Optika i Spektroskopiya 88(3), 495-498 (2000).

18. J. F. Nye, Natural Focusing and the Fine Structure of Light, Institute of Physics Publishing, Bristol (1999).

19. J. F. Nye, "Polarization effects in the diffraction of electromagnetic waves: The role of disclinations," Proc. R. Soc. A 387, 105-132 (1983).

20. O. V. Angelsky, A. Y. Bekshaev, P. P. Maksimyak, A. P. Maksimyak, I. I. Mokhun, S. G. Hanson, C. Y. Zenkova, A. V. Tyurin, "Circular motion of particles suspended in a Gaussian beam with circular polarization validates the spin part of the internal energy flow," Opt. Express 20(10), 11351-11356 (2012).

21. J. F. Nye, J. V. Hajnal, "The wave structure of monochromatic electromagnetic radiation," Proc. R. Soc. A 409, 21-36 (1987).

22. M. V. Berry, M. R. Dennis, "Polarization singularities in isotropic random vector waves," Proc. R. Soc. A 457, 141-155 (2001).

23. A. D. Dolgov, A. G. Doroshkevich, D. I. Novikov, I. D. Novikov, "Classification of singular points in polarization field of cosmic microwave background and eigenvectors of Stokes matrix," JETP Lett. 69, 427-433 (1999).

24. A. I. Konukhov, L. A. Melnikov, "Optical vortices in a vector field: the general definition based on the analogy with topological solitons in a 2D ferromagnet, and examples from the transverse polarization patterns of lasers," J. Opt. B: Quantum Semiclass. Opt. 3, S139-S144 (2001).

25. O. V. Angelsky, A. Y. Bekshaev, P. P. Maksimyak, A. P. Maksimyak, S. G. Hanson, C. Y. Zenkova, "Orbital rotation without orbital angular momentum: mechanical action of the spin part of the internal energy flow in light beams," Opt. Express 20(4), 3563-3571 (2012).

26. A. Y. Bekshaev, O. V. Angelsky, S. G. Hanson, C. Y. Zenkova, "Scattering of inhomogeneous 
Yu. A. Ushenko, G. B. Bodnar \& G. D. Koval

circularly polarized optical field and mechanical manifestation of the internal energy flows," Phys. Rev. A 86, 023847 (2012).

27. Freund, "Coherency matrix description of optical polarization singularities," J. Opt. A.: Pure and Appl. Opt. 6, S229-S234 (2004).
28. A. G. Ushenko, V. P. Pishak, Laser polarimetry of biological tissue: Principles and applications, Handbook of Coherent-Domain Optical Methods: Biomedical Diagnostics, Environmental and Material Science, Vol. 1, V. V. Tuchin, Ed., pp. 93-138, Kluwer Academic Publishers (2004). 